\title{
Prognostic Subcellular Notch2, Notch3 and Jagged1 Localization Patterns in Early Triple-negative Breast Cancer
}

\author{
TITIKA-MARINA STRATI ${ }^{1}$, VASSILIKI KOTOULA ${ }^{2,3}$, IOANNIS KOSTOPOULOS ${ }^{2}$, \\ KYRIAKI MANOUSOU ${ }^{4}$, CHRISTOS PAPADIMITRIOU ${ }^{5}$, GEORGIOS LAZARIDIS ${ }^{6}$, SOTIRIS LAKIS $^{3}$, \\ GEORGE PENTHEROUDAKIS ${ }^{7}$, DIMITRIOS PECTASIDES ${ }^{8}$, ELISSAVET PAZARLI ${ }^{9}$, \\ CHRISTOS CHRISTODOULOU ${ }^{10}$, EVANGELIA RAZIS ${ }^{11}$, KITTY PAVLAKIS $^{12}$, CHRISTINA MAGKOU ${ }^{13}$, \\ SOFIA CHRISAFI ${ }^{3}$, GERASIMOS ARAVANTINOS ${ }^{14}$, DIMITRIOS BAFALOUKOS ${ }^{15}$, PAVLOS PAPAKOSTAS ${ }^{16}$, \\ HELEN GOGAS ${ }^{17}$, KONSTANTINE T. KALOGERAS ${ }^{3,18}$ and GEORGE FOUNTZILAS ${ }^{3,19}$ \\ ${ }^{1}$ Third Department of Surgery, AHEPA University Hospital, Aristotle University of Thessaloniki, \\ School of Health Sciences, Faculty of Medicine, Thessaloniki, Greece; \\ ${ }^{2}$ Department of Pathology, Aristotle University of Thessaloniki, \\ School of Health Sciences, Faculty of Medicine, Thessaloniki, Greece; \\ ${ }^{3}$ Laboratory of Molecular Oncology, Hellenic Foundation for Cancer Research/ \\ Aristotle University of Thessaloniki, Thessaloniki, Greece; \\ ${ }^{4}$ Section of Biostatistics, Hellenic Cooperative Oncology Group, Data Office, Athens, Greece; \\ ${ }^{5}$ Department of Clinical Therapeutics, Alexandra Hospital, \\ National and Kapodistrian University of Athens School of Medicine, Athens, Greece; \\ ${ }^{6}$ Department of Medical Oncology, Papageorgiou Hospital, Aristotle University of Thessaloniki, \\ School of Health Sciences, Faculty of Medicine, Thessaloniki, Greece; \\ ${ }^{7}$ Ioannina University Hospital, Ioannina, Greece; \\ ${ }^{8}$ Oncology Section, Second Department of Internal Medicine, Hippokration Hospital, Athens, Greece; \\ ${ }^{9}$ Department of Pathology, Papageorgiou Hospital, Aristotle University of Thessaloniki, \\ School of Health Sciences, Faculty of Medicine, Thessaloniki, Greece; \\ ${ }^{10}$ Second Department of Medical Oncology, Metropolitan Hospital, Piraeus, Greece; \\ ${ }^{11}$ Third Department of Medical Oncology, Hygeia Hospital, Athens, Greece; \\ ${ }^{12}$ Pathology Department, National and Kapodistrian University of Athens Medical School, Athens, Greece; \\ ${ }^{13}$ Pathology Department, Evangelismos Hospital, Athens, Greece; \\ ${ }^{14}$ Second Department of Medical Oncology, Agii Anargiri Cancer Hospital, Athens, Greece; \\ ${ }^{15}$ First Department of Medical Oncology, Metropolitan Hospital, Piraeus, Greece; \\ ${ }^{16}$ Oncology Unit, Hippokration Hospital, Athens, Greece; \\ ${ }^{17}$ First Department of Medicine, Laiko General Hospital, National and Kapodistrian \\ University of Athens School of Medicine, Athens, Greece; \\ ${ }^{18}$ Translational Research Section, Hellenic Cooperative Oncology Group, Data Office, Athens, Greece; \\ ${ }^{19}$ Aristotle University of Thessaloniki, Thessaloniki, Greece
}

\begin{abstract}
Background: The Notch pathway has been implicated in triple-negative breast cancer $(T N B C)$. Herein, we studied the subcellular localization of the less investigated

Correspondence to: Titika-Marina Strati, MD, MSc, 3rd Surgical Department, AHEPA University Hospital, Thessaloniki, Greece. Tel: +306972525009, Fax: +30 2106912713, e-mail: Titika_marina_strati@ mail.com
\end{abstract}

Key Words: Notch2, Notch3, Jagged1, triple-negative breast cancer, prognosis.
Notch2 and Notch3 and that of the Jaggedl (Jagl) ligand in patients with operable TNBC. Patients and Methods: We applied immunohistochemistry for Notch2, Notch3 and Jag1 in 333 tumors from TNBC patients treated with adjuvant anthracycline-based chemotherapy. We evaluated cytoplasmic $(c)$, membranous $(m)$ and nuclear $(n)$ protein localization. Results: c-Notch2 (35\% positive tumors), c-Notch3 (63\%), cJag1 (43\%), m-Notch3 (23\%) and n-Jag1 (17\%) were analyzed individually and by using hierarchical clustering for prognostic evaluation. Upon multivariate analysis, compared to high $m$-Notch3 in the absence of n-Jagl (cluster 4), all other marker combinations (clusters 1, 2, 3) conferred 
significantly higher risk for relapse $(p<0.05)$. Conclusion: Specific Notch3 and Jag1 subcellular localization patterns may provide clues for the behavior of the tumors and potentially for Jagl targeting in TNBC patients.

Triple-negative breast cancer (TNBC) accounts for 10-20\% of all breast cancers (1). TNBC shows a poor outcome, with a high rate of local and systemic relapse and shorter survival, probably because of more aggressive biological behavior, but also because of lack of effective treatments $(2,3)$. Because TNBC tumors lack ER, PgR and HER2 receptor expression they do not respond to targeted treatment agents, such as tamoxifen and trastuzumab (4). Various types of neoadjuvant chemotherapies and targeted therapies are investigated in this context, some of which are focusing on promising targets, such as components of the Notch signaling pathway, a molecular cascade of critical importance for cell proliferation, differentiation, development and homeostasis (5).

The Notch pathway involves four receptors, Notch1 to Notch4 and five cell-bound ligands, DLL1, DLL2, DLL3, Jagged1 (Jag1) and Jagged2 (Jag2); Notch receptors undergo extensive cleavage modifications upon ligand binding and form intracellular Notch complexes that translocate to the nucleus to function as transcription activators (6). The Notch pathway has been implicated in multiple forms of cancer and multiple aspects of cancer biology, including tumor angiogenesis, neoplastic cell growth, cancer stem cell maintenance and metastatic processes (6-8). The contribution of aberrant Notch signaling to breast cancer was first noticed in the murine mammary gland (9) and then in human breast cancer cell lines. Elevated expression of Notch1 and/or Jag1 in human breast tumors was associated with poor overall patient survival (1012), with basal phenotype (12-14) and increased expression of survivin, an apoptotic cell death inhibitor, particularly in ERnegative and basal cancers (13-15). Notch1 and Notch4 are expressed in TNBC (16), with Notch4 being proposed as a therapeutic target (17). Overall, although Notch1, Notch4 and Jag1 are considered to be oncogenic in breast cancer, with multiple inhibition strategies being proposed (8), little is known about the role of Notch2, Notch3 and their ligands, particularly with respect to their subcellular localization in tumor cells, which would reflect their functional status $(6,8)$.

Herein, in a series of patients with operable TNBC and complete clinical data, we examined protein expression and subcellular localization of the less studied Notch receptors, Notch2 and Notch3, along with the Jag1 ligand and compared their expression patterns with patient outcome. We also evaluated possible associations between the above molecules with protein expression of E- and P-cadherin, based on the recently proposed down-regulation of cell adhesion by Notch signaling (18), and with protein expression of $\mathrm{p} 53$, based on the previously reported association of p53 with Jag1 (12).

\section{Patients and Methods}

Patient cohort. The present study was conducted retrospectively in a cohort of patients with TNBC. The translational research study was approved by the Bioethics Committee of the Aristotle University of Thessaloniki, School of Medicine (June 28, 2013). All patients included in the study provided written informed consent for the use of their biological material for future research.

In total, 418 cases, reported as TNBC, were retrieved from the patient database of the Hellenic Cooperative Oncology Group; patients had participated in 6 prospective breast cancer adjuvant trials (HE10/97, HE10/00, HE10/04, HE10/05, HE10/08 and HE10/10) between 1997 and 2013. Except for the first trial, formalin-fixed paraffin-embedded (FFPE) tumor tissue blocks were prospectively collected. All above clinical trials investigated the efficacy of anthracycline-based treatment regimens in the management of operable breast cancer patients. Baseline characteristics and clinical outcomes of the first four trials have already been described (19-22).

Tumors were initially characterized as TNBC in local pathology laboratories with ER, PgR and HER2 immunohistochemistry. Tumor histology and TNBC phenotype were re-evaluated centrally, as previously described (23). The latter involved ER, PgR and HER2 immunohistochemistry and fluorescence in situ hybridization (FISH) for HER2 gene status where indicated. Eighty-five cases were excluded on the basis of unavailable FFPE tissue or discordant central typing; 333 patients with centrally validated TNBC were finally included in this study (Figure 1).

Tissue microarray (TMA) construction and immunohistochemistry (IHC). Tissue processing and IHC were performed at the Laboratory of Molecular Oncology of the Hellenic Foundation of Cancer Research/Aristotle University of Thessaloniki. Histological evaluation was performed by an experienced pathologist (S.L.), who reviewed hematoxylin-eosin (H\&E)-stained slides from tissue blocks for adequacy of material and calculation of the percentage of tumor cells in each case. Two tissue cores per tumor, $1.5 \mathrm{~mm}$ in diameter, were arrayed in TMAs with a manual arrayer (Model I, Beecher Instruments, San Prairie, WI, USA), as previously described $(24,25)$. Serial 3- $\mu \mathrm{m}$ thick sections from the TMA blocks were mounted on adhesive microscope slides and subjected to IHC staining in Bond $\operatorname{Max}^{\mathrm{TM}}$ (Leica Microsystems, Wezlar, Germany) and 16000 (Biogenex, San Ramon, CA, USA) autostainers. We studied Notch2, Notch3 and Jag 1 protein expression for cytoplasmic, nuclear and membranous localization. We used the following antibodies and respective conditions: Notch2 (code 72803, polyclonal, Abcam, 20' EDTA, at 1:100 dilution for 30 minutes, Bond polymers kit), Notch3 (code 23426, polyclonal, Abcam, 20' EDTA at 1:500 dilution for 30 min, Bond polymers kit) and Jag1 (code HPA021555, polyclonal, Sigma Aldrich, 20' citric acid at dilution 1:125 overnight, Bond polymers kit). We also applied IHC for p53 (clone DO-7, code M7001, Dako, 20' citric acid, at 1:100 dilution for $20 \mathrm{~min}$, Bond polymers kit), E-cadherin (clone 36B5, cat No PA0387, RTU, Leica Biosystems, 20' EDTA, RTU, Bond protocol F), P-cadherin (clone 56C1, code M1741, Thermo Scientific, 20' EDTA, 1:200-O/N, Bond polymers kit) and Ki67 (clone MIB-1, code M7240, Dako, 20' EDTA, 1:70-20', Bond polymers kit). The antigen-antibody complex was visualized with diaminobenzidine (DAB) as a chromogen. Slides were counterstained with Mayer's hematoxylin, washed in fresh water, dehydrated and mounted. 


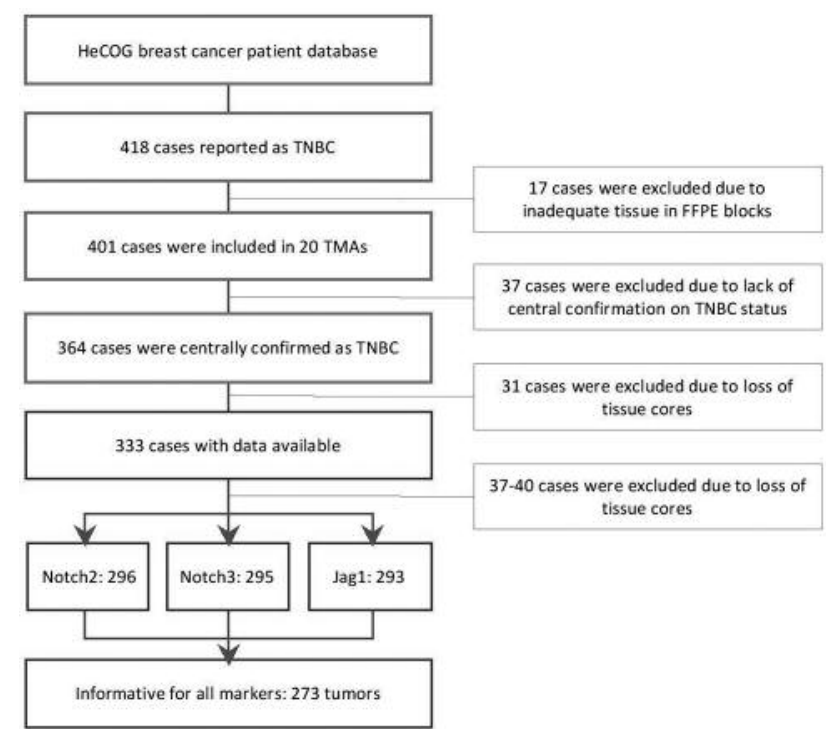

Figure 1. REMARK diagram.

As external controls, we used cores (10 in total for each TMA) from various non-neoplastic and neoplastic tissues, including placental, endometrial, thyroid, kidney, tonsil, mammary gland, prostate, melanoma and papillary carcinoma of thyroid. As internal controls, we used the neighboring stroma of mammary gland lobules, as well as the normal, hyperplastic and dysplastic epithelium.

Tumor lymphocytic infiltrations were assessed in a qualitative manner as a 4-scale variable: whole H\&E sections were screened for lymphocytic infiltrations in the immediate proximity with cancer cells; at least 5 areas with the most dense stromal infiltrations were evaluated under 200X magnification; tumors were classified as negative or 0 ( 0 to $\leq 1 \%$ lymphocytic infiltration); weak or 1 ( $>1 \%$ to $25 \%)$; intermediate or 2 (>25\% to $75 \%)$; high or $3(>75 \%)$. Because this classification differs from the widely applied stromal tumor infiltrating lymphocytes (TILs) density, we do not use the term TILs for the herein used classification; however, the context is the same.

IHC evaluation and scoring system. For Notch2, Notch3 and Jag1 intensity and positivity rates were evaluated. Regarding the cutoffs, staining intensity was scored into four grades $(0,1+, 2+$ and $3+)$, while the percentage of positive cells was also scored into four categories ( 0 for $0 \%, 1$ for $1-33 \%, 2$ for $>33-66 \%$ and 3 for $>66$ $100 \%)(10)$. The product of the intensity and percentage categories was used as the final score (26) and classified as negative (0-4) and positive (5-9). Positivity for E-cadherin, P-cadherin and p53 was considered for values of $\geq 10 \%$ at any intensity (27). Ki67 was used as a continuous variable (average percentage of positive nuclei). Similarly, all other study markers were also used as continuous measurements of percentage positivity. Lymphocytic infiltrations were assessed on a 4-level scale, as described above, and as a binary variable for high $(>75 \%)$ versus all other categories.

Statistical analysis. Categorical variables were presented as counts and corresponding percentages, while various measures were used for the continuous variables (mean, standard deviation, median, min, $\max$ ), as well as histograms and box-plots. The Fisher's exact or
Pearson chi-square tests were used for group comparisons of categorical data, while for continuous data the non-parametric Mann-Whitney or Kruskal-Wallis tests were used where appropriate. Correlations among continuous markers were assessed by Pearson's $r$ or Spearman's Rho.

Disease-free survival (DFS) was measured from the date of diagnosis until verified disease progression or death, whichever occurred first (28), while overall survival (OS) from diagnosis until death from any cause. Patients alive or progression-free were censored at the date of last contact. Survival curves were estimated using the Kaplan-Meier method and compared across groups with log-rank tests. Univariate Cox regression analyses were used to examine prognostic significance of the study parameters and report their effects (hazard ratios, HR) on DFS and OS.

Model choice was performed using backward selection criteria with a removal criterion $p=0.15$, including in the initial step the following clinical parameters: age ( $<$ median $v s$. $\geq$ median in years), tumor size ( $\leq 2 v s .2-5 v s .>5 \mathrm{~cm})$, histological grade (I-II vs. III), number of positive nodes ( $0 v s . \geq 4,1-3 v s . \geq 4)$, adjuvant hormonal therapy (yes $v s$. no), adjuvant radiotherapy (yes $v s$. no) and Ki67 percent (as a continuous variable).

In addition, unsupervised hierarchical clustering, using the Ward's minimum variance method, was conducted for IHC markers with continuous measurements, i.e., Notch2, Notch3, Jag1 and Ki67 in order to identify distinct groups with common biological characteristics. Scatter-plots and box-plots were used for the description of the clusters. The final clusters included the markers that allowed for better cluster discrimination. The optimal number of clusters was selected by the use of pseudo F-statistics (ratio of between-cluster variance to within-cluster variance, a measure of how separated the clusters were).

The statistical analyses were performed using the SAS software (SAS for Windows, version 9.3, SAS Institute Inc., Cary, NC, USA). Statistical significance was set at 2 -sided $p=0.05$. Results of this study were presented according to reporting recommendations for tumor marker prognostic studies (29). This study is prospective/retrospective as described in Simon et al. (30).

\section{Results}

Approximately half the patients were postmenopausal; almost equal numbers of patients had been treated with breast-conserving surgery and modified radical mastectomy (MRM); most tumors were of high histological grade (grade III) and of the non-specific (NOS) type, followed by medullary, lobular, metaplastic and apocrine histologies. Patient and tumor characteristics are summarized in Table I.

Notch2, Notch3 and Jag1 as single IHC markers. Notch2, Notch3 and Jag1 proteins were assessed for cytoplasmic, membranous and nuclear staining that was classified as positive or negative. These binary IHC results along with examples of the main subcellular localization patterns are shown in Figure 2. Cytoplasmic Notch2 immunopositivity was found in $35.4 \%$ of the tumors; membranous and nuclear staining was not observed for this protein. More than half of the tumors exhibited cytoplasmic Notch3 immunopositivity; for this protein, membranous positivity was observed in 
$23.1 \%$ of tumors and nuclear staining was absent. Jag1 was localized in the cytoplasm $(42.7 \%)$ and nucleus $(17.1 \%)$, with only four tumors exhibiting membranous staining. As shown in Supplemental Table I, tumors negative for cytoplasmic Notch3 were more frequent in older women (59.3\%, Fisher's exact test, $p=0.022)$; all tumors with nuclear Jag1 $(p<0.001)$ and the vast majority of those with cytoplasmic and membranous Notch3 $(91.9 \%, p<0.001$ and $95.5 \%, p=0.006$, respectively) and cytoplasmic Notch2 (78.1\%, $p=0.045)$ were of the basal-like phenotype; tumors positive for cytoplasmic Notch3, membranous Notch3 and nuclear Jag1 had higher median Ki67 protein expression (Mann-Whitney, $p$-values $\leq 0.001$ ), whereas tumors positive for cytoplasmic Jag1 had lower median Ki67 expression $(p<0.001)$.

The rates of positive $v s$. negative tumors were $59.1 \% \mathrm{vs}$. $40.9 \%$ for p53 protein expression (informative tumors $\mathrm{n}=308), 71.3 \%$ vs. $28.7 \%$ for E-cadherin $(\mathrm{n}=296)$ and $83.2 \%$ vs. $16.8 \%$ for P-cadherin $(\mathrm{n}=309)$, respectively; as can be deduced, up to $10 \%$ of the tumors were noninformative for each of these three markers. As shown in Supplemental Table II, basal-like tumors were predominantly positive for both cadherins (Fisher's exact, $p$-values $<0.001)$ and for $\mathrm{p} 53$ protein $(p=0.015)$. There was also an association of these three markers with histological type ( $p$-values <0.005). More specifically, tumors of the non-specific type were more often positive for these markers, while protein positivity in these markers was associated with higher median Ki67 expression (MannWhitney, $p$-values $\leq 0.001)$.

Correlations between Notch2, Notch3, Jag1, E-cadherin, P-cadherin, p53, and Ki67 expression are shown in Supplemental Table III. Except for the strong positive correlation between E- and P-cadherin (Spearman's Rho 0.621 ), as previously described (27), weaker positive correlations were observed between cytoplasmic and membranous Notch3 (Rho 0.321), and also between cytoplasmic Notch3 and Ki67 labeling (Rho 0.364); cytoplasmic Jag1 was inversely correlated with Ki67 (Rho 0.340 ) and with nuclear Jag1 (Rho -0.362).

No associations were observed between cadherins and Notch markers, either as categorical or continuous variables, probably because the interaction between these molecular systems appears to be indirectly mediated through Slug (31); similarly, p53 protein expression was not related to the examined Notch parameters. For these reasons, and also because of the considerable amount of missing data for protein expression of cadherins and p53, these markers were excluded from further analyses.

Hierarchical clustering of continuous IHC measurements. Because there are no consensus cutoffs for Notch2, Notch3 and Jag1, since these proteins have only been examined in research settings, and also because they are co-expressed in
Table I. Patient demographics and standard clinicopathological characteristics.

\begin{tabular}{|c|c|c|}
\hline Patients (N=333) & Category/metrics & $\mathrm{N}(\%)$ \\
\hline \multirow[t]{7}{*}{ Origin } & Breast Registry & $38(11.4)$ \\
\hline & HE10/97 & $19(5.7)$ \\
\hline & HE10/00 & $85(25.5)$ \\
\hline & HE10/04 & $21(6.3)$ \\
\hline & HE10/05 & $87(26.1)$ \\
\hline & HE10/08 & $72(21.6)$ \\
\hline & HE10/10 & $11(3.3)$ \\
\hline \multirow[t]{2}{*}{ Adjuvant hormonal therapy } & Yes & $64(19.2)$ \\
\hline & No & $269(80.8)$ \\
\hline \multirow[t]{2}{*}{ Adjuvant radiotherapy } & Yes & $244(73.2)$ \\
\hline & No & $89(26.8)$ \\
\hline \multirow[t]{2}{*}{ Age (median cutoff) } & $<53.2$ & $166(49.9)$ \\
\hline & $\geq 53.2$ & $167(50.1)$ \\
\hline \multirow[t]{3}{*}{ Basal phenotype } & Yes & $271(81.4)$ \\
\hline & No & $55(16.6)$ \\
\hline & Not reported & $7(2.2)$ \\
\hline \multirow[t]{4}{*}{ Breast surgery } & Biopsy only & $5(1.5)$ \\
\hline & Breast conserving surgery & $169(50.8)$ \\
\hline & MRM & $152(45.6)$ \\
\hline & Unknown & $7(2.1)$ \\
\hline \multirow[t]{6}{*}{ Histological type } & Apocrine & $9(2.7)$ \\
\hline & Lobular & $18(5.4)$ \\
\hline & Medullar & $21(6.3)$ \\
\hline & Metaplastic & $17(5.1)$ \\
\hline & Non-specific & $257(77.2)$ \\
\hline & Other & $11(3.3)$ \\
\hline \multirow[t]{3}{*}{ Histological grade } & I-II & $59(17.7)$ \\
\hline & III & $271(81.4)$ \\
\hline & Not reported & $3(0.9)$ \\
\hline \multirow{5}{*}{$\begin{array}{l}\text { Lymphocytic infiltrates } \\
\text { (whole sections) }\end{array}$} & $0(\leq 1 \%)$ & $141(42.3)$ \\
\hline & $1(>1-25 \%)$ & $82(24.6)$ \\
\hline & $2(26-75 \%)$ & $56(16.8)$ \\
\hline & $3(>75 \%)$ & 39 (11.7) \\
\hline & Not reported & $15(4.6)$ \\
\hline \multirow{3}{*}{$\begin{array}{l}\text { Lymphocytic infiltrates } \\
\text { (Low vs. High) }\end{array}$} & Low & $279(83.8)$ \\
\hline & High $(>75 \%)$ & $39(11.7)$ \\
\hline & Not reported & $15(4.5)$ \\
\hline \multirow[t]{3}{*}{ Menopausal status } & Premenopausal & $144(43.2)$ \\
\hline & Postmenopausal & $181(54.4)$ \\
\hline & Unknown & $8(2.4)$ \\
\hline \multirow[t]{2}{*}{ Multifocal } & No & $313(94)$ \\
\hline & Yes & $20(6)$ \\
\hline \multirow[t]{4}{*}{ Number of positive nodes } & 0 & $107(32.1)$ \\
\hline & $1-3$ & $116(34.8)$ \\
\hline & $\geq 4$ & $101(30.3)$ \\
\hline & Not reported & $9(2.8)$ \\
\hline \multirow[t]{4}{*}{ Tumor size } & $\leq 2 \mathrm{~cm}$ & 115 (34.6) \\
\hline & $2-5 \mathrm{~cm}$ & $184(55.2)$ \\
\hline & $>5 \mathrm{~cm}$ & $28(8.4)$ \\
\hline & Not reported & $6(1.8)$ \\
\hline \multirow[t]{3}{*}{ Ki67 labeling } & $\mathrm{N}$ & 317 \\
\hline & Median & 46.5 \\
\hline & Range & $0-100$ \\
\hline
\end{tabular}

N, Number; MRM, modified radical mastectomy. 
A
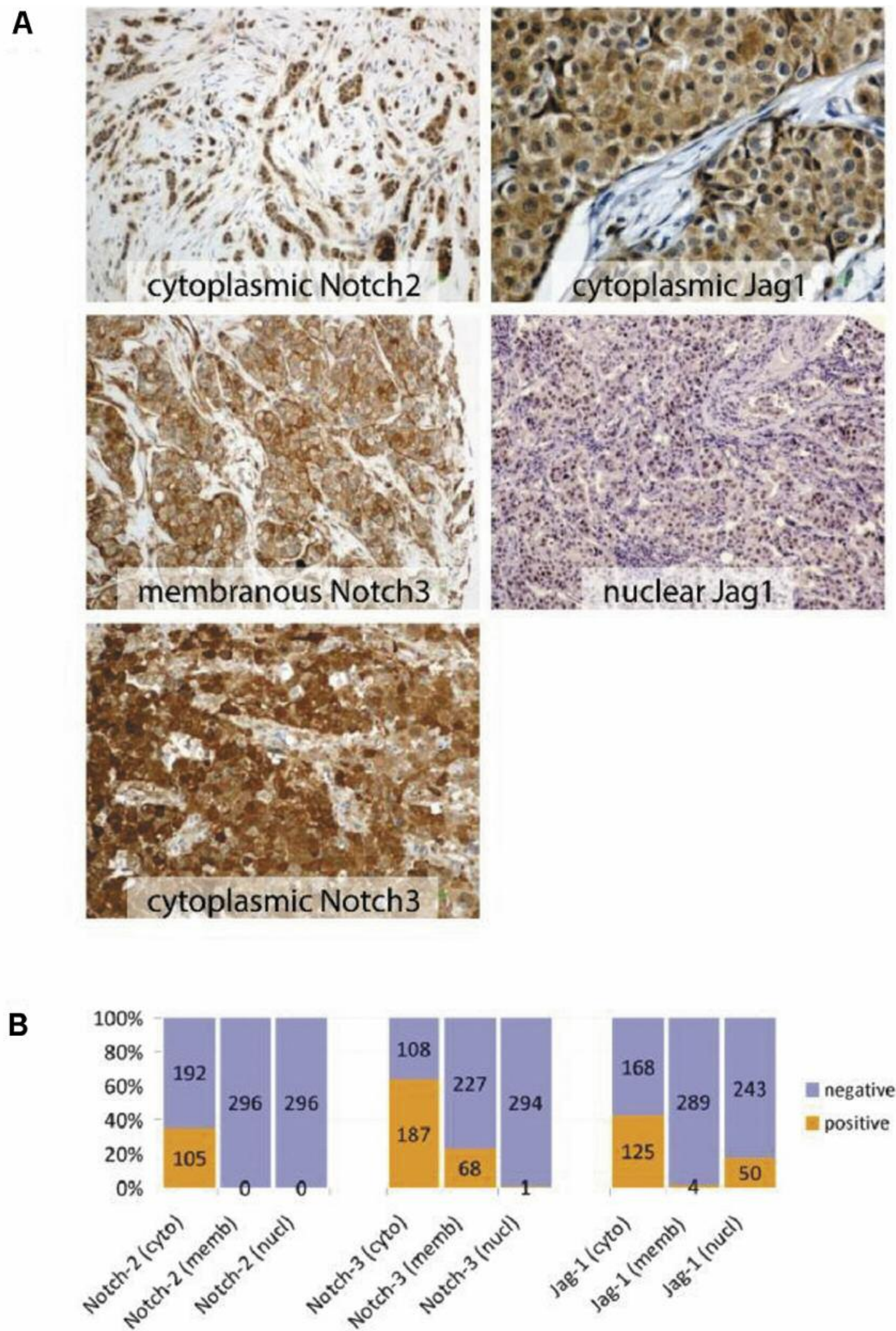

Figure 2. Subcellular localization patterns of Notch-related proteins in TNBC. A. Immunohistochemical markers and localization as indicated. Green bars: $10 \mu \mathrm{m}$. In the tumor with cytoplasmic Jag1 staining, faint membranous staining was also present. B. Distribution of subcellular localization patterns for Notch2, Notch3 and Jag1 in the examined tumors. 


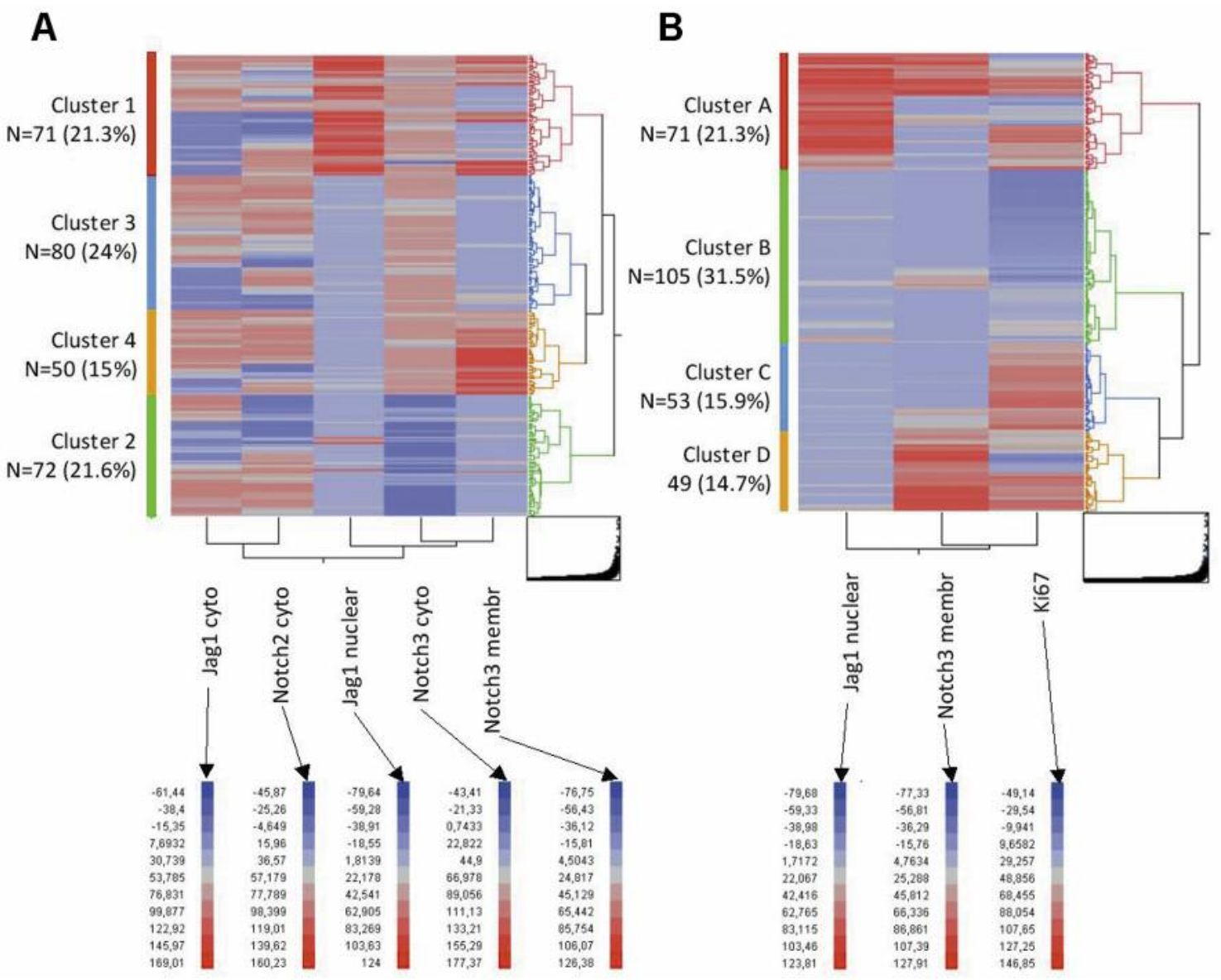

Figure 3. Hierarchical clustering of continuous IHC measurements for the assessment of Notch localization patterns. A: All Jag/Notch clusters included all evaluable Notch-related localization markers. B: Dominant markers from model (A), nuclear Jag1 and membranous Notch3 were clustered with Ki67. In both cases clusters were well separated, as inferred from the distanced dendrograms. Note the similarity with respect to membranous Notch 3 and nuclear Jag1 between clusters 1 and A, 2 and B, 3 and C, 4 and D.

Table II. Multivariate COX regression analysis adjusting the effects of all Jag/Notch clusters on DFS.

\begin{tabular}{|c|c|c|c|c|c|}
\hline DFS $(N=262)$ & $\mathrm{N}$ of patients & $\mathrm{N}$ of events & HR & $95 \% \mathrm{CI}$ & Wald's $\mathrm{p}$ \\
\hline \multicolumn{6}{|l|}{ Age (median cutoff) } \\
\hline$\geq 53.2 \mathrm{vs} .<53.2$ & 133 vs. 129 & 37 vs. 45 & 0.69 & $0.44-1.08$ & 0.101 \\
\hline \multicolumn{6}{|l|}{ Number of positive nodes } \\
\hline $0 v s . \geq 4$ & $88 v s .82$ & 18 vs. 44 & 0.31 & $0.17-0.57$ & $<0.001$ \\
\hline $1-3 v s . \geq 4$ & 92 vs. 82 & 20 vs. 44 & 0.28 & $0.16-0.49$ & $<0.001$ \\
\hline \multicolumn{6}{|l|}{ Histological grade } \\
\hline III $v s$. I-II & $220 v s .42$ & 74 vs. 8 & 2.32 & $1.08-5.01$ & 0.032 \\
\hline \multicolumn{6}{|l|}{ Adjuvant HT } \\
\hline Yes $v s$. No & 48 vs. 214 & 22 vs. 60 & 1.68 & $1.01-2.80$ & 0.044 \\
\hline \multicolumn{6}{|l|}{ Adjuvant RT } \\
\hline Yes vs. No & 198 vs.64 & 58 vs. 24 & 0.41 & $0.24-0.70$ & 0.001 \\
\hline \multicolumn{6}{|l|}{ All Jag/Notch clusters } \\
\hline Cluster 1 vs. Cluster 4 & $66 v s .49$ & 23 vs. 7 & 2.41 & $1.02-5.71$ & 0.046 \\
\hline Cluster 2 vs. Cluster 4 & 69 vs. 49 & $26 v s .7$ & 2.45 & $1.04-5.77$ & 0.040 \\
\hline Cluster 3 vs. Cluster 4 & 78 vs. 49 & $26 \mathrm{vs} .7$ & 2.61 & $1.13-6.05$ & 0.025 \\
\hline
\end{tabular}

N, Number; HR, hazard ratio; CI, confidence interval; HT, hormonal therapy; RT, radiotherapy. 

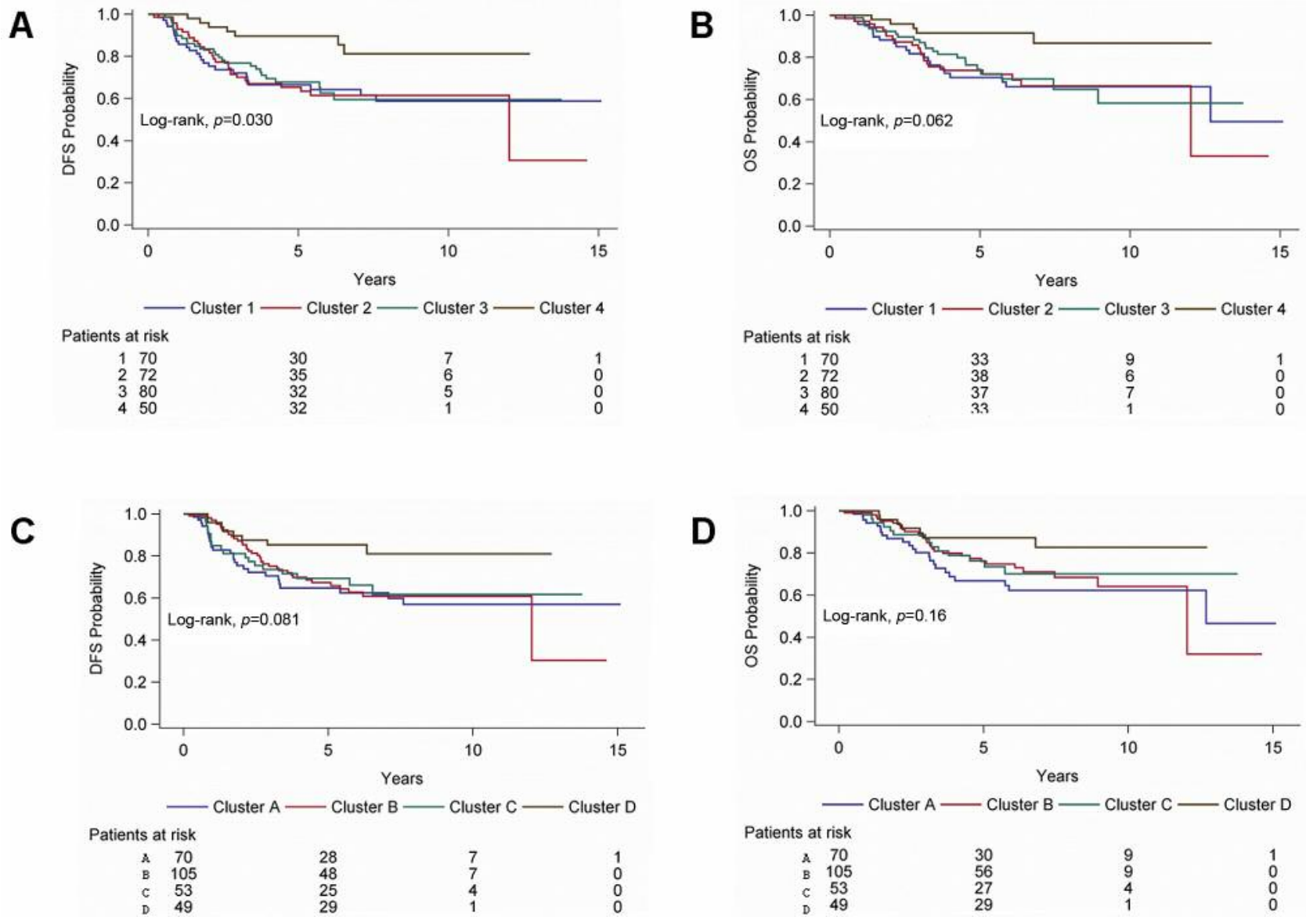

Figure 4. Effect of membranous Notch3 and nuclear Jag1 on patient outcome. A and B: All Jag/Notch clusters; C and D: Jag/Notch/Ki67 clusters. Note the constant superior outcome in patients with cluster 4 and cluster D tumors.

the same tumor and hence, they are expected to function in tandem, we next used continuous measurements for the Notch-related IHC markers in hierarchical clustering models. Since 4 of the 9 markers were not expressed in all (membranous and nuclear Notch2) or almost all (nuclear Notch3 and membranous Jag1) of the patients, these markers were not analyzed. Thus, analyses were only performed for the cytoplasmic Notch2, cytoplasmic Notch3, membranous Notch3, cytoplasmic Jag1 and nuclear Jag1 markers.

Based on the predicted best separation, 4 clusters were distinguished for all Jag/Notch markers (Figure 3A): cluster 1 , with tumors overexpressing nuclear Jag1 with various combinations of the rest of the markers; cluster 2, with complete absence of Notch3 and Jag1 expression; cluster 3, with absence of membranous Notch3 and nuclear Jag1; and, cluster 4, with strong membranous Notch3 but without nuclear Jag1. Cytoplasmic Notch2 and cytoplasmic Jag1 were randomly dispersed among clusters. Because of this pattern, the two markers were removed from the models. In addition, as described above, all markers except for cytoplasmic Jag1 were positively correlated with Ki67 (Supplemental Table III). Thus, we next clustered the markers that appeared to be dominant, i.e., membranous Notch3 and nuclear Jag1 with Ki67. These clusters (Figure 3B) were named alphabetically as follows: cluster A, with nuclear Jag1 overexpression; cluster B, negative for membranous Notch3, nuclear Jag1 and Ki67; cluster C, with high Ki67 but negative for the Notch3 and Jag1 markers; and, cluster D, with strong membranous Notch3 and high Ki67 expression but negative for nuclear Jag1. When comparing both clustering models (Figure 3), similar features were observed for clusters 1 and A (high nuclear Jag1), as well as clusters 4 and D (high membranous Notch3).

As shown in Supplemental Table IV, basal-like phenotype was significantly associated with the clusters in both models (Fisher's exact, $p$-values <0.001). More specifically, basallike tumors were more often represented in clusters 1 (29.6\%) and 3 (29.6\%), as well as clusters A (29.3\%) and B 
(31\%). There was also a significant association between the clusters in both models and histological type $(p=0.001$ for clusters 1, 2, 3, 4 and $p=0.019$ for clusters A, B, C, D). More specifically, medullary carcinomas were more frequent in clusters $4(52.9 \%)$ and D (47\%). In the same line, lymphocytic infiltrates were significantly associated with clusters in both clustering models ( $p=0.006$ for clusters 1,2 , 3,4 and $p=0.013$ for clusters A, B, C, D); among tumors with a high rate $(>75 \%)$ of lymphocytic infiltrates, $40.0 \%$ was observed in cluster 4 and $34.3 \%$ in cluster D. Finally, Ki67 expression was significantly different among the four clusters in both models (Kruskal-Wallis, $p$-values <0.001). More specifically, cluster 2 that was negative for membranous Notch3 and nuclear Jag1 had significantly lower median Ki67.

Association of all study markers with patient outcome. The median follow-up of the cohort was 75.9 months (range 2.3181.1). The median DFS and OS were not reached. Overall, 80 patients $(24 \%)$ died and 98 relapses occurred $(29.4 \%)$. DFS at 3 years was $77.6 \%$ and at 5 years $72.2 \%$. OS at 3 years was $87.2 \%$ and at 5 years $77.9 \%$. The estimated effects of all studied parameters on DFS and OS are shown in Supplemental Tables V and VI, respectively. High nodal status and larger tumor size were unfavorable prognosticators for both DFS and OS (Wald's, $p$-values <0.001), as expected.

When examined separately, none of the study markers was significantly associated with DFS or OS (Supplemental Tables V and VI). A trend towards improved DFS was observed in patients with positive membranous Notch3 tumors $(\mathrm{HR}=0.61,95 \% \mathrm{CI}=0.35-1.06$, Wald's $p=0.082)$, while a trend towards improved OS was observed in patients with positive cytoplasmic Notch $2(\mathrm{HR}=0.64,95 \% \mathrm{CI}=0.38$ 1.07, $p=0.089$ ).

Clustered markers significantly predicted for outcome, with clusters 4 and D conferring favorable prognosis. No relapses at 3 years were observed for $89.6 \%$ of the patients with tumors expressing high membranous Notch3 but no nuclear Jag1 (cluster 4); in comparison, $70.1 \%$ of patients with tumors lacking nuclear Jag1 and any Notch3 expression (cluster 2), $72.1 \%$ of those expressing high nuclear Jag1 (cluster 1) and $76.8 \%$ of those expressing cytoplasmic Notch3 (cluster 3) were event free at 3 years (Figure 4A). DFS at 5 years for patients in cluster 4 was $89.6 \%$, while it was $65.4 \%$ for cluster 2, 66.5\% for cluster 1 and $67.8 \%$ for cluster 3 (log-rank, $p=0.030$ ). Patients with cluster 3 tumors had significantly higher risk for relapse as compared to patients with cluster 4 tumors $(\mathrm{HR}=2.91,95 \% \mathrm{CI}=1.27-6.69$, Wald's $p=0.012$ ) (Supplemental Table V). The favorable prognostic significance for cluster 4 as compared to cluster 3 was also evident for OS (Figure 4B), with cluster 4 tumors conferring lower risk for death (cluster 3 vs. $4, \mathrm{HR}=3.07$, 95\%CI=1.16-8.12, $p=0.024$ ) (Supplemental Table VI).
Nuclear Jag1 and membranous Notch3 expression combined with Ki67 yielded similar effects on outcome, as described for the all Jag/Notch clusters (Figure 4C and D). Patients with cluster D tumors, expressing high membranous Notch3 but no nuclear Jag1, performed better as compared to those with cluster A tumors overexpressing nuclear Jag1 [cluster A vs. D for DFS: $\mathrm{HR}=2.73,95 \% \mathrm{CI}=1.24-6.04$, Wald's $p=0.013$ (Supplemental Table V); for OS: HR=2.59, 95\%CI $=1.11-6.050, p=0.028$ (Supplemental Table VI)].

Clustered markers were adjusted for all standard clinicopathological parameters in two separate models. The favorable prognostic impact of cluster 4 against clusters 1,2 and 3 retained its significance only for DFS (Table II). The favorable prognostic impact of cluster D against clusters A and $\mathrm{C}$ was not retained in the multivariate setting.

All Supplementary Tables are publicly available at: http://hecog-images.gr/TNBC_NOTCH_SUPPLEMENTAL_ TABLES/

\section{Discussion}

We studied the expression and subcellular localization of three members of the Notch pathway, receptors Notch2 and Notch3 and ligand Jag1, in a patient cohort with operable TNBC treated with standard adjuvant chemotherapy. We showed that these factors are expressed with distinct localization in TNBC tumor samples and present a novel, potentially clinically-relevant Notch3/Jag 1 expression pattern, as a favorable marker in this heterogeneous group of cancers. In order to interpret the latter finding though, we need to discuss the importance of the subcellular localization of Notch receptors and ligands.

A particular characteristic of the Notch pathway is that both receptors and ligands in adjacent cells are membranebound molecules, which must be cleaved by proteases in order to produce their effects; the first cleavage step for Notch receptors is by gamma-secretase $(5,6,32)$. As described in these reviews, detection of these molecules on the cell membrane possibly reflects a mature but inactivated state; in the cytoplasm, potentially an initially activated state upon cleavage; and in the nucleus, the active state. All of the IHC antibodies we used bind to the intracellular part of Notch2, Notch3 and Jag1 and are expected to detect the cleaved intracellular domains of these proteins in the cytoplasm and in the nucleus. Our findings of frequent cytoplasmic but absent nuclear Notch2 and Notch3 expression thus indicates that these receptors may have undergone initial activation but failed to be fully activated in order to exert canonical Notch effects, i.e., transcriptional regulation. Cytoplasmic Notch2 was associated with low nodal burden and non-basal-like phenotype, indicating lack of full activation of this molecule. In addition, we detected Notch3 on tumor cell membranes in about $20 \%$ of the 
examined TNBC, which may be interpreted as being an inactive state. Experimental evidence suggests that Notch3 may inhibit the cell cycle by inducing cell adhesion with CDH1 (33) and suppress tumor growth by inducing cell senescence (34), indicating antitumor effects for this molecule. Thus, although cytoplasmic Notch3 was associated with aggravating tumor features, such as basal-like phenotype, high proliferation rate and high nodal burden, the overall IHC pattern of Notch3 was not compatible with a tumorigenic role in TNBC, in line with the above studies (33, 34). Based on their localization patterns, Notch2 and Notch3 did not appear to function as transcriptional regulators in the present TNBC series and, although they were associated with contrasting clinicopathological characteristics, they did not individually affect patient outcome, as also suggested by a study on Notch mRNA expression in ER-positive and ERnegative breast cancers (35).

Like Notch receptors, Notch ligands are also cleaved upon communicating with adjacent cells; (5) for example, in myocardial cells and upon further cleavage, Jag1 intracellular domain molecules may translocate to the nucleus and induce maturation in the neonate or protect from hypertrophy in the adult (36). In addition, cleaved extracellular Notch1 can be internalized in the ligand cell and interfere with Jag1 translocation to the nucleus (37). Herein, we observed cytoplasmic localization of Jag1 in about $40 \%$ of our TNBC patients, consistent with both functional variations described above, and nuclear Jag1 in a smaller part of these tumors, all of them of the immunohistochemichally determined basal-like phenotype. Jag1 mRNA has been linked to the basal phenotype (12), Jag $1 \mathrm{mRNA}$ and protein were associated with worse breast cancer prognosis $(10,11)$ and fluorescently determined Jag1 was described as a marker of aggressive breast carcinomas, also present in the circulating cells shed by these tumors (38). Our findings are in line with these reports and they further indicate that nuclear Jag1 localization may be related to the reported aggravating role of this protein in breast carcinomas; the molecular context of nuclear Jag1 function and effects needs to be elucidated.

Apparently, the studied proteins and their localization patterns were co-expressed in tumors. Hence, it was important to observe whether these interacted with each other for the outcome of patients upon conventional adjuvant cytotoxic chemotherapy. We showed that tumors with inactive Notch3, as indicated by membranous Notch3 protein expression, in the absence of nuclear Jag1, conferred favorable prognosis in the given clinical context. This finding has two aspects: (a) it highlights the importance of assessing and reporting the functional status of Notch pathway components, as indicated by their subcellular localization; and, (b) it indicates that tumors with expressed but apparently inactive Notch3 may benefit from cytotoxic treatment. With respect to the latter, inhibition of active Notch with gamma secretase inhibitors (39) or with specific anti-Notch1 targeting agents (40) results in sensitization of breast cancers to docetaxel, through depletion of cancer stem cells. The same mechanistic frame may apply to our patients with membranous Notch3/no-nuclear Jag1 tumors, i.e., naïve Notch3 inactive, who appeared to benefit from anthracycline-based chemotherapy.

Here, high membranous Notch3 protein expression, presumably inactive, was effective as a favorable prognosticator in the absence of nuclear Jag1 but not in its presence. This pattern implies that Notch3 may in fact be inhibited by Jag1, in accordance with the above described Notch inhibition by nuclear Jag1 in a preclinical model (36). Of note, the favorable membranous Notch3 did not seem to have a growth-suppressive impact, as experimentally suggested (34), since most of the tumors in this group had a high proliferation rate, which as a single parameter was not associated with treatment benefit. Thus, out of all parameters examined, the dominant one in TNBC appears to be nuclear Jag1. Ligand cleavage necessitates different targeting than the gamma-secretase described above (6); such drugs might be tested in TNBC $(6,41)$.

Importantly, the favorable membranous Notch3/no-nuclear Jag1 tumors were rich in lymphocytic infiltrations and included half of the medullary carcinomas in the cohort. Lymphocytic infiltrations were not assessed according to the widely accepted recommendations for stromal TILs density (42), which may be the reason for failure of this marker to reach statistical prognostic significance in the present study. However, since dense lymphocytic infiltrations are a known favorable prognostic factor for TNBC patients in the adjuvant setting $(43,44)$, it is tempting to speculate that, at least in part, this parameter contributed to the favorable prognosis of patients with high membranous Notch3/nonuclear Jag1 tumors. Whether the Notch-related features of these tumors contributed to the attraction of immune cells in the tumor microenvironment is currently unknown. In the only relevant study so far showing that Notch up-regulation attracts FOXP3-positive Tregs into the tumor (45), Notch3 was not examined. Given the established role of Notch receptors in the maintenance of breast cancer stem cells (46), and the fact that cancer stem cells in general need to escape host immune surveillance (47), it can be speculated that active Notch would not facilitate host anti-tumor immunity. A possible scenario might then be that inactive Notch, particularly Notch3 in our case, would permit the accumulation of lymphocytic infiltrates in the tumor. In any case, the herein observed Notch3/Jag1 patterns are new findings and should be considered as hypothesis generating, however they seem worthy pursuing for preclinical validation and larger translational studies, especially in view of the current trend for immunotherapeutic interventions. 
In view of the already trialed gamma secretase inhibitors in TNBC though, the herein observed inactive or semiactivated Notch2 and Notch3 in TNBC are in line with published observations that most members of the Notch pathway are active, and hence worthy targeting, in the presence of ER but not in its absence $(35,45)$. As previously published, the role of Notch in solid tumors appears to be dependent on the molecular context (5); overexpression of Notch members in breast cancer may not always be progrowth and pro-dissemination (6), while there are effects by members of the Notch pathway that do not result from gamma-secretase cleavage (non-canonical) and would therefore not be inhibited by the corresponding drugs (8), like the nuclear Jag1 effects described earlier. Although a limitation of the present study is that we did not evaluate all members of the pathway, our results are in full concordance with the above-mentioned reports. And, as previously suggested (32), we once more emphasize how important it is to assess subcellular Notch receptor and ligand localization patterns for obtaining a view of the molecular context reflecting the activation status of this pathway. Notch expression is not synonymous with its active state; it seems plausible that only patients with active Notch receptors will benefit from relative targeting, and they therefore need to be identified among patients with TNBC.

In conclusion, we showed that two receptors of the Notch pathway, Notch2 and Notch3, might not be canonically activated, while the Jag1 ligand seems to be activated in about $17 \%$ of operable TNBC. Specific Notch3 and Jag1 subcellular localization patterns may provide clues for the behavior of the corresponding tumors and could potentially be applied in the clinic for Jag1 targeting in TNBC patients. However, given the prospective/retrospective design of our study, our findings should be confirmed in prospective validation cohorts.

\section{Acknowledgements}

The Authors are indebted to all patients and their families for their trust and participation in the Hellenic Cooperative Oncology Group trials and for the provision of biological material for research purposes.

\section{References}

1 Brenton JD, Carey LA, Ahmed AA and Caldas C: Molecular classification and molecular forecasting of breast cancer: Ready for clinical application? J Clin Oncol 23(29): 7350-7360, 2005.

2 Carey LA, Perou CM, Livasy CA, Dressler LG, Cowan D, Conway K, Karaca G, Troester MA, Tse CK, Edmiston S, Deming SL, Geradts J, Cheang MC, Nielsen TO, Moorman PG, Earp HS and Millikan RC: Race, breast cancer subtypes, and survival in the carolina breast cancer study. JAMA 295(21): 2492-2502, 2006

3 Irvin WJ Jr. and Carey LA: What is triple-negative breast cancer? Eur J Cancer 44(18): 2799-2805, 2008.
4 Lehmann BD and Pietenpol JA: Identification and use of biomarkers in treatment strategies for triple-negative breast cancer subtypes. J Pathol 232(2): 142-150, 2014.

5 Ranganathan $\mathrm{P}$, Weaver KL and Capobianco AJ: Notch signalling in solid tumours: A little bit of everything but not all the time. Nat Rev Cancer 11(5): 338-351, 2011.

6 Ntziachristos P, Lim JS, Sage J and Aifantis I: From fly wings to targeted cancer therapies: A centennial for notch signaling. Cancer Cell 25(3): 318-334, 2014.

7 Li D, Masiero M, Banham AH and Harris AL: The notch ligand jagged1 as a target for anti-tumor therapy. Front Oncol 4: 254, 2014.

8 Previs RA, Coleman RL, Harris AL and Sood AK: Molecular pathways: Translational and therapeutic implications of the notch signaling pathway in cancer. Clin Cancer Res 21(5): 955-961, 2015.

9 Gallahan D and Callahan R: Mammary tumorigenesis in feral mice: Identification of a new int locus in mouse mammary tumor virus (czech ii)-induced mammary tumors. J Virol 61(1): 66-74, 1987.

10 Reedijk M, Odorcic S, Chang L, Zhang H, Miller N, McCready DR, Lockwood G and Egan SE: High-level coexpression of jag1 and notch1 is observed in human breast cancer and is associated with poor overall survival. Cancer Res 65(18): 8530-8537, 2005.

11 Dickson BC, Mulligan AM, Zhang H, Lockwood G, O'Malley FP, Egan SE and Reedijk M: High-level jag1 mrna and protein predict poor outcome in breast cancer. Mod Pathol 20(6): 685693, 2007.

12 Reedijk M, Pinnaduwage D, Dickson BC, Mulligan AM, Zhang H, Bull SB, O'Malley FP, Egan SE and Andrulis IL: Jag1 expression is associated with a basal phenotype and recurrence in lymph node-negative breast cancer. Breast Cancer Res Treat 111(3): 439-448, 2008.

13 Lee CW, Raskett CM, Prudovsky I and Altieri DC: Molecular dependence of estrogen receptor-negative breast cancer on a notch-survivin signaling axis. Cancer Res 68(13): 5273-5281, 2008.

14 Lee CW, Simin K, Liu Q, Plescia J, Guha M, Khan A, Hsieh CC and Altieri DC: A functional notch-survivin gene signature in basal breast cancer. Breast Cancer Res 10(6): R97, 2008.

15 van Es JH, van Gijn ME, Riccio O, van den Born M, Vooijs M, Begthel H, Cozijnsen M, Robine S, Winton DJ, Radtke F and Clevers H: Notch/gamma-secretase inhibition turns proliferative cells in intestinal crypts and adenomas into goblet cells. Nature 435(7044): 959-963, 2005.

16 Speiser J, Foreman K, Drinka E, Godellas C, Perez C, Salhadar A, Ersahin C and Rajan P: Notch-1 and notch-4 biomarker expression in triple-negative breast cancer. Int J Surg Pathol 20(2): 139-145, 2012.

17 Nagamatsu I, Onishi H, Matsushita S, Kubo M, Kai M, Imaizumi A, Nakano K, Hattori M, Oda Y, Tanaka M and Katano M: Notch4 is a potential therapeutic target for triplenegative breast cancer. Anticancer Res 34(1): 69-80, 2014.

18 Chen J, Imanaka N, Chen J and Griffin JD: Hypoxia potentiates notch signaling in breast cancer leading to decreased e-cadherin expression and increased cell migration and invasion. $\mathrm{Br} \mathrm{J}$ Cancer 102(2): 351-360, 2010.

19 Fountzilas G, Dafni U, Papadimitriou C, Timotheadou E, Gogas H, Eleftheraki AG, Xanthakis I, Christodoulou C, Koutras A, Papandreou CN, Papakostas P, Miliaras S, Markopoulos C, 
Dimitrakakis C, Korantzopoulos P, Karanikiotis C, Bafaloukos D, Kosmidis P, Samantas E, Varthalitis I, Pavlidis N, Pectasides D and Dimopoulos MA: Dose-dense sequential adjuvant chemotherapy followed, as indicated, by trastuzumab for one year in patients with early breast cancer: First report at 5-year median follow-up of a hellenic cooperative oncology group randomized phase iii trial. BMC Cancer 14: 515, 2014.

20 Fountzilas G, Pectasides D, Christodoulou C, Timotheadou E, Economopoulos T, Papakostas P, Papadimitriou C, Gogas H, Efstratiou I and Skarlos D: Adjuvant dose-dense sequential chemotherapy with epirubicin, cmf, and weekly docetaxel is feasible and safe in patients with operable breast cancer. Med Oncol 23(4): 479-488, 2006.

21 Fountzilas G, Skarlos D, Dafni U, Gogas H, Briasoulis E, Pectasides D, Papadimitriou C, Markopoulos C, Polychronis A, Kalofonos HP, Siafaka V, Kosmidis P, Timotheadou E, Tsavdaridis D, Bafaloukos D, Papakostas P, Razis E, Makrantonakis P, Aravantinos G, Christodoulou C and Dimopoulos AM: Postoperative dose-dense sequential chemotherapy with epirubicin, followed by $\mathrm{cmf}$ with or without paclitaxel, in patients with high-risk operable breast cancer: A randomized phase iii study conducted by the hellenic cooperative oncology group. Ann Oncol 16(11): 1762-1771, 2005.

22 Gogas H, Dafni U, Karina M, Papadimitriou C, Batistatou A, Bobos M, Kalofonos HP, Eleftheraki AG, Timotheadou E, Bafaloukos D, Christodoulou C, Markopoulos C, Briasoulis E, Papakostas P, Samantas E, Kosmidis P, Stathopoulos GP, Karanikiotis C, Pectasides D, Dimopoulos MA and Fountzilas G: Postoperative dose-dense sequential versus concomitant administration of epirubicin and paclitaxel in patients with nodepositive breast cancer: 5-year results of the hellenic cooperative oncology group he 10/00 phase iii trial. Breast Cancer Res Treat 132(2): 609-619, 2012.

23 Fountzilas G, Dafni U, Bobos M, Batistatou A, Kotoula V, Trihia H, Malamou-Mitsi V, Miliaras S, Chrisafi S, Papadopoulos S, Sotiropoulou M, Filippidis T, Gogas H, Koletsa T, Bafaloukos D, Televantou D, Kalogeras KT, Pectasides D, Skarlos DV, Koutras A and Dimopoulos MA: Differential response of immunohistochemically defined breast cancer subtypes to anthracycline-based adjuvant chemotherapy with or without paclitaxel. PLoS One 7(6): e37946, 2012.

24 Kononen J, Bubendorf L, Kallioniemi A, Barlund M, Schraml P, Leighton S, Torhorst J, Mihatsch MJ, Sauter G and Kallioniemi OP: Tissue microarrays for high-throughput molecular profiling of tumor specimens. Nat Med 4(7): 844-847, 1998.

25 Skacel M, Skilton B, Pettay JD and Tubbs RR: Tissue microarrays: A powerful tool for high-throughput analysis of clinical specimens: A review of the method with validation data. Appl Immunohistochem Mol Morphol 10(1): 1-6, 2002.

26 Allred DC, Harvey JM, Berardo M and Clark GM: Prognostic and predictive factors in breast cancer by immunohistochemical analysis. Mod Pathol 11(2): 155-168, 1998.

27 Lakis S, Dimoudis S, Kotoula V, Alexopoulou Z, Kostopoulos I, Koletsa T, Bobos M, Timotheadou E, Papaspirou I, Efstratiou I, Aravantinos G, Karavasilis V, Zagouri F, Gogas H, Razis E, Pentheroudakis G, Christodoulou C, Pectasides D and Fountzilas G: Interaction between beta-catenin and egfr expression by immunohistochemistry identifies prognostic subgroups in early high-risk triple-negative breast cancer. Anticancer Res 36(5): 2365-2378, 2016.
28 Hudis CA, Barlow WE, Costantino JP, Gray RJ, Pritchard KI, Chapman JA, Sparano JA, Hunsberger S, Enos RA, Gelber RD and Zujewski JA: Proposal for standardized definitions for efficacy end points in adjuvant breast cancer trials: The steep system. J Clin Oncol 25(15): 2127-2132, 2007.

29 McShane LM, Altman DG, Sauerbrei W, Taube SE, Gion M, Clark GM and Statistics Subcommittee of NCIEWGoCD: Reporting recommendations for tumor marker prognostic studies (remark). Breast Cancer Res Treat 100(2): 229-235, 2006.

30 Simon RM, Paik S and Hayes DF: Use of archived specimens in evaluation of prognostic and predictive biomarkers. J Natl Cancer Inst 101(21): 1446-1452, 2009.

31 Shao S, Zhao X, Zhang X, Luo M, Zuo X, Huang S, Wang Y, $\mathrm{Gu} S$ and Zhao $\mathrm{X}$ : Notch1 signaling regulates the epithelialmesenchymal transition and invasion of breast cancer in a slugdependent manner. Mol Cancer 14: 28, 2015.

32 Speiser JJ, Ersahin C and Osipo C: The functional role of notch signaling in triple-negative breast cancer. Vitam Horm 93: 277306, 2013.

33 Chen CF, Dou XW, Liang YK, Lin HY, Bai JW, Zhang XX, Wei XL, Li YC and Zhang GJ: Notch3 overexpression causes arrest of cell cycle progression by inducing cdh 1 expression in human breast cancer cells. Cell Cycle 15(3): 432-440, 2016.

$34 \mathrm{Cui} \mathrm{H}$, Kong Y, Xu M and Zhang $\mathrm{H}$ : Notch3 functions as a tumor suppressor by controlling cellular senescence. Cancer Res 73(11): 3451-3459, 2013.

$35 \mathrm{Xu} \mathrm{J}$, Song F, Jin T, Qin J, Wu J, Wang M, Wang Y and Liu J: Prognostic values of notch receptors in breast cancer. Tumour Biol 37(2): 1871-1877, 2016.

36 Metrich M, Bezdek Pomey A, Berthonneche C, Sarre A, Nemir $M$ and Pedrazzini T: Jagged1 intracellular domain-mediated inhibition of notch1 signalling regulates cardiac homeostasis in the postnatal heart. Cardiovasc Res 108(1): 74-86, 2015.

37 Hansson EM, Lanner F, Das D, Mutvei A, Marklund U, Ericson J, Farnebo F, Stumm G, Stenmark H, Andersson ER and Lendahl $\mathrm{U}$ : Control of notch-ligand endocytosis by ligand-receptor interaction. J Cell Sci 123(Pt 17): 2931-2942, 2010.

38 Bednarz-Knoll N, Efstathiou A, Gotzhein F, Wikman H, Mueller $\mathrm{V}$, Kang Y and Pantel K: Potential involvement of jagged1 in metastatic progression of human breast carcinomas. Clin Chem 62(2): 378-386, 2016.

39 Schott AF, Landis MD, Dontu G, Griffith KA, Layman RM, Krop I, Paskett LA, Wong H, Dobrolecki LE, Lewis MT, Froehlich AM, Paranilam J, Hayes DF, Wicha MS and Chang JC: Preclinical and clinical studies of gamma secretase inhibitors with docetaxel on human breast tumors. Clin Cancer Res 19(6): 1512-1524, 2013.

40 Qiu M, Peng Q, Jiang I, Carroll C, Han G, Rymer I, Lippincott J, Zachwieja J, Gajiwala K, Kraynov E, Thibault S, Stone D, Gao Y, Sofia S, Gallo J, Li G, Yang J, Li K and Wei P: Specific inhibition of notch1 signaling enhances the antitumor efficacy of chemotherapy in triple negative breast cancer through reduction of cancer stem cells. Cancer Lett 328(2): 261-270, 2013.

41 Yuan X, Wu H, Xu H, Xiong H, Chu Q, Yu S, Wu GS and Wu $\mathrm{K}$ : Notch signaling: An emerging therapeutic target for cancer treatment. Cancer Lett 369(1): 20-27, 2015.

42 Salgado R, Denkert C, Demaria S, Sirtaine N, Klauschen F, Pruneri G, Wienert S, Van den Eynden G, Baehner FL, PenaultLlorca F, Perez EA, Thompson EA, Symmans WF, Richardson AL, Brock J, Criscitiello C, Bailey H, Ignatiadis M, Floris G, 
Sparano J, Kos Z, Nielsen T, Rimm DL, Allison KH, Reis-Filho JS, Loibl S, Sotiriou C, Viale G, Badve S, Adams S, WillardGallo K, Loi S and International TWG: The evaluation of tumorinfiltrating lymphocytes (tils) in breast cancer: Recommendations by an international tils working group 2014. Ann Oncol 26(2): 259-271, 2015.

43 Kotoula V, Chatzopoulos K, Lakis S, Alexopoulou Z, Timotheadou E, Zagouri F, Pentheroudakis G, Gogas H, Galani E, Efstratiou I, Zaramboukas T, Koutras A, Aravantinos G, Samantas E, Psyrri A, Kourea H, Bobos M, Papakostas P, Kosmidis P, Pectasides D and Fountzilas G: Tumors with highdensity tumor infiltrating lymphocytes constitute a favorable entity in breast cancer: A pooled analysis of four prospective adjuvant trials. Oncotarget 7: 5074-5087, 2016.

44 Loi S, Michiels S, Salgado R, Sirtaine N, Jose V, Fumagalli D, Kellokumpu-Lehtinen PL, Bono P, Kataja V, Desmedt C, Piccart MJ, Loibl S, Denkert C, Smyth MJ, Joensuu H and Sotiriou C: Tumor infiltrating lymphocytes are prognostic in triple negative breast cancer and predictive for trastuzumab benefit in early breast cancer: Results from the finher trial. Ann Oncol 25(8): 1544-1550, 2014.
45 Ortiz-Martinez F, Gutierrez-Avino FJ, Sanmartin E, PomaresNavarro E, Villalba-Riquelme C, Garcia-Martinez A, Lerma E and Peiro G: Association of notch pathway down-regulation with triple negative/basal-like breast carcinomas and high tumorinfiltrating foxp3+ tregs. Exp Mol Pathol 100(3): 460-468, 2016.

46 Rangel MC, Bertolette D, Castro NP, Klauzinska M, Cuttitta F and Salomon DS: Developmental signaling pathways regulating mammary stem cells and contributing to the etiology of triplenegative breast cancer. Breast Cancer Res Treat 156(2): 211-226, 2016.

47 Schumacher TN and Schreiber RD: Neoantigens in cancer immunotherapy. Science 348(6230): 69-74, 2015.

Received January 12, 2017

Revised March 26, 2017

Accepted April 4, 2017 